\title{
INNOVACIÓN EN LA ACTIVIDAD TURÍSTICA VASCA: HACIA UN MODELO COMPETITIVO EN LA C.A. de EUSKADI
}

\author{
Aurkene Alzua Sorzabal ${ }^{1}$ \\ CICtourGUNE \\ Marina Abad Galzacorta ${ }^{2}$ \\ Universidad de Deusto
}

\begin{abstract}
Resumen:
En un marco global de creciente importancia económica de la industria turística, los datos atestiguan que la evolución del sector turístico en la Comunidad Autónoma de Euskadi muestra una senda de crecimiento sostenido, que sin embargo viene marcado por un cambio progresivo en la estructura de la demanda y por un entorno donde cada vez hay más competencia. La adecuación del sistema a esta nueva realidad y en consecuencia, a las nuevas necesidades, constituye un importante reto desde el punto de vista de la gestión turística y pasa, necesariamente, por la mejora de la competitividad del turismo vasco. Por otro lado, el papel central de la innovación en los distintos procesos económicos y en la mejora de la competitividad de un país es irrefutable y además existen evidencias de que la innovación es el factor dominante de la competitividad internacional, y por tanto, del crecimiento económico nacional. En definitiva, en el nuevo entorno competitivo en el que se desarrolla la actividad turística mundial, parece que la adopción y el uso estratégico de las TIC pueden ayudar a la empresa a mejorar su eficiencia y a ser más competitiva, esencialmente a través del canal que supone el dinamismo innovador. En este estudio se ha realizado un acercamiento a esta realidad vasca con el objetivo de entender mejor cómo se está situando el sector turístico vasco en este nuevo entorno competitivo, es decir, conocer mejor cómo se está llevando a cabo esa adopción estratégica de innovación y de TIC, tratando igualmente de identificar cuáles son las principales barreras en la adopción de la innovación.
\end{abstract}

Palabras clave: turismo, competitividad, innovación, Comunidad Autónoma de Euskadi, regresión lineal.

1 Aurkene Alzua Sorzabal, CICtourGUNE, Mikeletegi Pasealekua, 56 - 201, Parque Tecnológico de San Sebastián, 20009 Donostia-San Sebastián. Tfno: 943.010.885, e-mail: aurkenealzua@tourgune.org

2 Marina Abad Galzacorta, Universidad de Deusto, Mundaiz, 50, 20012 Donostia-San Sebastián.Tfno: 943.326.600, e-mail:1maabad@rigel.deusto.es 
Resumo: Innovación na actividade turística vasca:cara a un modelo competitivo na Comunidade Autónoma de Euskadi.

Nun marco global de crecente importancia económica da industria turística, os datos testemuñan que a evolución do sector turístico en Euskadi amosa unha senda de crecemento sostido que, no entanto, vén marcado por un cambio progresivo na estrutura da demanda e por un contorno en que cada vez hai máis competencia. A adecuación do sistema a esta nova realidade e, en consecuencia, ás novas necesidades, constitúe un importante reto dende o punto de vista da xestión turística e pasa, necesariamente, pola mellora da competitividade do turismo vasco. Por outro lado, o papel central da innovación nos distintos procesos económicos e na mellora da competitividade dun país é irrefutable: existen evidencias de que a innovación é o factor dominante da competitividade internacional e, por tanto, do crecemento económico nacional. En definitiva, no novo ámbito competitivo en que se desenvolve a actividade turística mundial, parece que a adopción e o uso estratéxico das TIC poden axudarlle á empresa a mellorar a súa eficiencia e a ser máis competitiva, esencialmente a través da canle que supón o dinamismo innovador. Neste estudo realizouse un achegamento á realidade vasca co obxectivo de entender mellor como se está a situar o sector turístico vasco neste novo ámbito competitivo, é dicir, coñecer mellor como se está a levar a cabo esa adopción estratéxica de innovación e de TIC, e tentouse, asemade, identificar cales son as principais barreiras na adopción da innovación que, sen dúbida, inciden nas competitividade do sector turístico vasco.

Palabras clave: turismo, competitividade, innovación, Euskadi, regresión lineal.

Abstract: Innovation in the Basque tourism activity: Towards a competitive model in the Autonomous Community of Euskadi.

Within a global framework of increasing economic weight of the tourism industry, the data show that the evolution of the tourism industry in the Autonomous Community of Euskadi follows a path of sustained growth, which, nevertheless, is marked by a progressive change in the structure of demand and by an increasingly competitive environment. Adaptation of the system to this new reality and, consequently, to the new needs, is a major challenge from the standpoint of tourism management and requires improving the competitiveness of Basque tourism. On the other hand, the major role of innovation in a country's different economic processes and improved competitiveness is irrefutable and, furthermore, there is evidence that innovation is the dominant factor in international competitiveness and, therefore, in national economic growth. Ultimately, in the new competitive environment in which world tourism activity takes place, it seems that the adoption and strategic use of ICT may help companies to improve their efficiency and become more competitive, essentially through the channels opened by innovative dynamism. In this study, we analyse this Basque reality in order to better understand how the Basque tourism industry is located within this new competitive environment; that is, to better understand how the strategic adoption of innovation and ICT is being carried out, while also attempting to identify the main barriers to the adoption of innovation, which irrefutably affect the competitiveness of the Basque tourism industry.

Key words: tourism, competitiveness, innovation, Autonomous Community of Euskadi, linear regression.

\section{INTRODUCCIÓN}

El significado económico y potencial de los Viajes y el Turismo (V\&T) se ha demostrado particularmente prominente tanto en economías avanzadas como en el mundo en desarrollo. Los Viajes y el Turismo ayudan a la diversificación de la actividad económica y al enriquecimiento del sector servicios de los países más avanzados, posibilitando así la creación de riqueza y puestos de trabajo tanto en áreas urbanas como en rurales. 
Entre 1950 y 2004, los ingresos del turismo internacional aumentaron de 2,1 billones a 622.7 billones de dólares, y para el año 2006, el sector de los V\&T fue responsable del 10,3 por ciento del PIB mundial. Ese mismo año, se contabilizaron 234 millones de empleos en la industria, constituyendo el 8,2 por ciento de los empleos mundiales. Es decir, la creciente importancia económica de la industria ha estado impulsada por el gran y ascendente número de viajeros internacionales (Blanke and Chiesa, 2007).

Comparablemente, los datos atestiguan que la evolución del sector turístico en la Comunidad Autónoma de Euskadi revela una senda de crecimiento sostenido, si bien, la evolución seguida desde 1997 muestra cambios radicales, tanto en dimensión como en la estructura del turismo vasco. Entre 1997 y 2004 se distinguen dos fases comprensiblemente diferenciadas (Plan de Competitividad del Turismo Vasco 2006-2009, 2006). La primera (1997-1999) caracterizada por el rápido crecimiento en el número de entradas de turistas y de pernoctaciones fundamentalmente en Bizkaia y más concretamente en Bilbao. Y una segunda fase, de consolidación, en la que se estabiliza dicho crecimiento con un nuevo arranque en 2004. Como refrendo de esta afirmación cabe destacar las altas tasas de crecimiento en la entrada de turistas registrados en establecimientos hoteleros en el periodo 1997-2004 (60\%), con un incremento ligeramente inferior del número de pernoctaciones (58\%); lo cual supone un crecimiento anual medio que se sitúa en el $10,4 \%$.

Según los datos procedentes de la Cuenta Satélite del Turismo de la C.A. de Euskadi 2000-2004, en el año 2004 el gasto turístico realizado en la C.A. de Euskadi alcanzó la cifra de 2.786 millones de euros, lo que significó una aportación de 5,2 \% al PIB. Más de la mitad del gasto turístico, el 55,6\%, fue generado por los visitantes (turistas y excursionistas) procedentes de fuera de la misma, mientras que el restante $44,4 \%$ del gasto se repartía entre el que hacen los turistas y los excursionistas residentes en la propia comunidad y el gasto en origen del turismo emisor. Más aún, ocurre que este periodo el crecimiento acumulativo anual supera el 6,2\% del PIB vasco. De esta forma, se confirma la tendencia suavemente creciente de la aportación del gasto turístico a la economía vasca.

Este avance del turismo vasco revela igualmente un cambio progresivo en la estructura de la demanda, que responde cada vez más a la motivación de ocio urbano complementada por el turismo de congresos y eventos. La adecuación del sistema a las necesidades de esta nueva estructura de la demanda constituye un importante reto desde el punto de vista de la gestión turística. Además del esfuerzo realizado, la competencia, cada vez mayor, requiere de un esfuerzo extra para mejorar la cuota de mercado y la rentabilidad de las empresas turísticas vascas. Esfuerzo que pasa, necesariamente, por la mejora de la competitividad del turismo vasco.

\section{CAMBIO DE PARADIGMA}

Las sociedades actuales están marcadas por la globalización y en este contexto la movilidad es un fenómeno en aumento que adquiere gran importancia. La movilidad 
implica contacto y transferencia de diversa naturaleza que integren lo social, lo económico y lo cultural. Esta transferencia toma especial relevancia en el ocio debido al aumento de tiempo libre en la sociedad del bienestar, como muestran los datos ofrecidos por la Organización Mundial del Turismo (OMT) referentes al 2006 que cifra en 880 millones los desplazamientos internacionales anuales.

La importancia del concepto de movilidad en la sociedad contemporánea también responde a la creciente centralidad que tienen los procesos de interconexión en la definición y funcionamiento de las dinámicas económicas y sociales del mundo contemporáneo (Castells, 2000). Consecuentemente, en esta sociedad de redes, la movilidad de personas, bienes e información, juegan un papel crucial en la formación de nuevos productos y servicios.

En este debate derivado de la movilidad, y su relación con el espacio y el tiempo, la actividad turística se ha considerado como determinante en la circulación y transferencia de flujos en su concepto más global, por su relación con la producción y el consumo de todo tipo de bienes. Es decir, la movilidad favorece nuevos encuentros, interacciones y patrones de producción de servicios así como nuevas formas de consumo.

En este contexto, hay que tener en cuenta que las distancias en el espacio y el tiempo son cada vez menos claras debido a la cercanía ofrecida por el desarrollo de las tecnologías. Sólo en el terreno físico el cambio tecnológico ha permitido ampliar las distancias que se pueden recorrer en una jornada. Consecuentemente, la frecuencia de los viajes y el volumen de bienes y personas que participan de estas oleadas de movilidad son uno de los fenómenos que caracterizan el momento presente. Por otro lado, en el plano de las no menos importantes movilidades virtuales o comunicativas, las transformaciones han sido también drásticas e innegables. Sobre este juego de reducción de la escala de tiempo y ampliación de lo abarcable en lo espacial, es indudable que la vida de las personas y de las sociedades contemporáneas se desarrolla bajo unos nuevos esquemas cuyas implicaciones todavía no son del todo comprendidas (Urry, 2000; Hannan et al., 2006).

En consonancia, las teorías sociales más recientes enfatizan la comprensión de las regiones o ciudades como lugares abiertos en el espacio y entrelazados por diferentes formas de movilidad, desde flujos de personas hasta flujos de bienes e información (Amin and Thrift, 2002). Los destinos turísticos se convierten, en ese sentido, en lugares de estacionamiento en el flujo constante de los flujos, movimientos e intercambios, mediados por las infraestructuras. Estos destinos, a su vez, también están dotados de un "metabolismo" de información que, aunque históricamente era de carácter principalmente económico (intercambio de bienes, compra-venta), ahora se componen de todo tipo de información, física y virtual, en constante circulación. Los nuevos intercambios e interacciones fruto de los flujos y las diversas movilidades, con las TIC como alimentadoras y reconfiguradoras, hacen que los espacios y sistemas móviles de la vida urbana y sus infraestructuras se tornen lugares estratégicos (Sheller and Urry, 2006).

Esta línea de pensamiento se corresponde con la literatura socio-económica reciente (Vilaseca et al., 2007; Castells, 1997; Garay, 2007), que identifica el uso y aplicación 
de las TIC como elemento medular del nuevo paradigma económico. Las TIC serían por tanto, la base material de la que se ha venido a llamar la tercera revolución industrial, que se caracteriza por la aplicación de conocimiento que a su vez genera nuevos conocimientos que a su vez, se extienden a todas las ramas de la actividad económica.

En este nuevo escenario económico y empresarial, los activos intangibles adquieren mayor protagonismo al situarse en el centro de los procesos productivos y de generación de conocimiento, y donde la innovación, entendida como la aplicación económica del conocimiento, se configura como condición necesaria para el desarrollo social y el crecimiento económico de un país o región (González y Peña, 2007; Morales y Peña, 2003).

Las exigencias de la vanguardia económica internacional y la plena inserción en la sociedad del conocimiento exigen la creación, desarrollo y consolidación de nuevos sectores intensivos en conocimiento. El avance hacia la sociedad del conocimiento implica que los sectores económicos de esa sociedad sean más intensivos en información para el desarrollo de sus actividades y, por lo tanto, necesiten disponer de nuevas herramientas que les capaciten para utilizar dicha información en todos los procesos implicados en la cadena de valor de la producción.

Este dinamismo se encuadra en un contexto de profundo cambio denominada por algunos autores como etapa posfordista (Calderón, 2007; Urry, 1990; Donaire, 1998). En este marco económico, donde el conocimiento es la pieza nuclear, es necesario apostar por un modelo en turismo que haga del conocimiento y de la innovación sus motores principales.

\section{COMPETITIVIDAD E INNOVACIÓN EN LA ACTIVIDAD TURÍSTICA}

El papel central de la innovación en los distintos procesos económicos y en la mejora de la competitividad de un país es irrefutable. Existe la evidencia de que la innovación es el factor dominante de la competitividad internacional, y por tanto, del crecimiento económico nacional. Así mismo la innovación es considerada como un factor que mejora la capacidad de una empresa para absorber y aprovechar nuevos conocimientos de todo tipo y por tanto, de mejorar y mantener su posición competitiva (OECD, 2005).

La globalización y el desarrollo tecnológico han generado en las empresas nuevas e importantes formas de acceso a la información. En particular, la evolución de las tecnologías de la información y la comunicación sumada a la convergencia tecnológica impacta en la mejora de la comunicación del conocimiento. Consecuentemente, la gestión y el uso del conocimiento se han extendido en toda la cadena de producción de valor y sirve para realizar tanto funciones de optimización y de control como para la toma de decisiones directivas. Por lo tanto, los avances tecnológicos y los flujos de información no sólo están transformando los productos y los precios sino también la naturaleza misma de la competencia (Porter, 2003).

En términos generales, la innovación se refiere a la asimilación y explotación exitosa de una idea para la mejora de procesos o introducción de nuevos productos o servicios en el mercado. Existen diversas metodologías aceptadas internacionalmente que 
proporcionan marcos conceptuales pertinentes para la medición de la actividad económica y social asociada a la innovación. Entre los documentos que suponen hoy una referencia pueden citarse el Manual de Frascatti (OECD, 95), el Manual de Canberra (OECD, 95) o el Manual de Oslo (OECD, 2005). Los citados informes reflejan el amplio y exhaustivo debate sobre la conceptualización de la innovación así como la relación de ésta con el desarrollo tecnológico y la actividad investigadora que se ha desarrollado durante algo más de la última década. Aunque no todos los resultados de la I+D son susceptibles de tener un uso práctico e incorporarse y convertirse en innovación, parece evidente la complementariedad e importancia del desarrollo tecnológico en la cadena de la producción de valor.

Por otro lado, y a pesar de las evidentes discrepancias, es necesario matizar que en el momento actual es muy difícil encontrar innovaciones que no estén de manera directa o transversalmente asociadas a usos nuevos de la tecnología. Por ello, a lo largo de este artículo se incluye, implícita o explícitamente, la innovación tecnológica cuando se habla de cualquier tipo de innovación. Así mismo y en lo concerniente a la propia conceptualización y medición de la innovación, cabe resaltar que pese a los grandes avances realizados, la medición y la comprensión de la innovación, sigue siendo preciso la ampliación de estudios y observaciones, debido en gran medida a la continua renovación del proceso de innovación asociado al dinamismo y desarrollo de la economía mundial.

Las teorías y tratados sobre innovación en lo concerniente al sector de servicios también han progresado notablemente, El Libro Verde hace mención explícita del sector servicios como "sector de innovación pero desconocido". La segunda edición del Manual de Oslo (1997) colocó el foco de estudio en las industrias fabriles, y no ha sido hasta su tercera edición (2005) en el que se ha establecido un nuevo marco con el fin de dar cabida a una amplia gama de industrias e innovaciones acordes con la realidad empresarial y económica del momento y entre las que se hallan las industrias de servicios (OECD, 2006).

Los incentivos y obstáculos que actúan en el desarrollo de las actividades innovadoras y su impacto son de gran importancia para entender el proceso de la innovación (OECD, 2005). Los objetivos y las barreras varían según el tipo de innovación. Por ejemplo, los objetivos de las innovaciones de producto o de marketing se refieren principalmente a la demanda mientras que las innovaciones de proceso y organizativas tratan más bien de la oferta. Las actividades de la innovación pueden ser obstaculizadas por diversos factores. En lo tocante a los principales obstáculos a la innovación, se incluyen razones para no iniciar en ningún caso actividades de innovación, factores que frenan las actividades de innovación o que tiene un efecto negativo sobre los resultados previstos. Puede tratarse de factores económicos, como sus elevados costes o una insuficiente demanda, de factores más vinculados a la demografía empresarial o bien a la falta de personal cualificado (Buhalis, 2003, 2006). Muchos de estos factores pueden ser conexos al tamaño de una empresa. El enfoque de "objeto" del Manual anteriormente citado, que incluye datos descriptivos, cuantitativos y cualitativos sobre una innovación en particular al mismo tiempo que recaba datos sobre la empresa (OCDE, 2006: 28), identifica el 
tamaño de la organización como un elemento esencial del diseño de las unidades estadísticas de las encuestas y recomienda medir el tamaño sobre la base de número de empleados. Esto es debido en parte, a que muchas actividades de innovación distintas de las de $\mathrm{I}+\mathrm{D}$ son muy comunes en las unidades de tamaño pequeño y mediano, por lo que se recomienda como mínimo las de diez empleados.

En la misma línea, existe literatura que presta especial atención al fenómeno del aumento considerable de las pequeñas y medianas empresas, como es el caso del tejido empresarial del sector turístico en Euskadi, y donde los resultados ponen de manifiesto que las empresas pequeñas son más dinámicas que las grandes en términos de crear empleo y que generan e introducen muchas innovaciones (Audretsch et al., 2002). En contraposición a esta tendencia, está empíricamente demostrado, por ejemplo, en términos de emprendimiento y coincidiendo con el estudio del Eurostat (2003), que cuanto menor es el tamaño de la empresa en el momento de su nacimiento menor es su probabilidad de supervivencia. Sin embargo y teniendo en cuenta que las nuevas empresas constituyen un colectivo heterogéneo, característica que también coincide con el sector turístico vasco, hay que prestar atención a la capacidad de gestión, la capacidad de innovación y la capacidad de internacionalización de las mismas, ya que éstas, dependen directamente de la cantidad y calidad de los recursos internos de la empresa (recursos humanos, recursos físicos y financieros) y también de los recursos externos que le proporciona su entorno económico como redes de cooperación o el acceso a mercados (Audretsch et al., 2002).

\subsection{LA INNOVACIÓN EN EL SECTOR TURISMO}

En cuanto la innovación dentro de la heterogeneidad del sector servicios, algunos estudios indican que la dinámica de la innovación en el sector del turismo tiende a ser incremental y que se corresponde generalmente con la incorporación de nuevos atributos o la mejora de los existentes, en respuesta al comportamiento del mercado y al interés por ganar cuota en el mismo. Frente a dicha apreciación lineal de la innovación, según Decelle (2004) el modelo que más se ajusta a la adopción de las TIC en la distribución turística es el del ciclo inverso (reverse cycle) formulado por Richard Barras (1986). Los estudios más recientes en materia de turismo y tecnologías de la información, sin embargo, parecen favorecer una tercera línea de innovación en la que conviven superpuestas diversas prácticas y existe una multiplicidad de modelos, primando en algunos casos las estrategias competitivas (Buhalis, 2003, 2006; Vilaseca et al., 2007; eBusinessWatch, 2007). Es importante añadir además que, dentro del sector servicios, coexisten actualmente algunas de las ramas más innovadoras de la actividad con aquellas que menos innovan (Evangelista y Savona, 1998).

Según el estudio realizado por los investigadores de la Universitat Oberta de Catalunya (Vilaseca et al. 2007) dentro del Proyecto Internet Catalunya (PIC) ${ }^{3}$, las empre-

3 El Proyecto Internet Catalunya (PIC) es un programa de investigación interdisciplinario sobre la sociedad de la información y el conocimiento en Catalunya realizado por investigadores del Internet Interdisciplinary Institute (IN3). 
sas turísticas con usos TIC más avanzados presentan una caracterización claramente diferenciada de la tradicional, aunque consideran que aún puede darse cuenta de la innovación en la empresa turística catalana desde una concepción lineal de la misma. Este estudio concluye que, en lo que concierne a la empresa turística catalana, la utilización de las TIC como elemento estratégico en la empresa está directamente relacionada con la presencia de mano de obra más cualificada, que trabaja con mayores y mejores equipamientos de Internet, lo que se deriva en una mayor capacidad para la generación y aplicación económica del conocimiento y, por lo tanto, en un mayor dinamismo innovador en la propia empresa. Además, las empresas con un uso más intensivo en TIC inician un cambio organizativo que les hace ser más flexibles, adaptar en mayor medida su oferta a las necesidades del mercado y subcontratar mejor sus operaciones. En paralelo se produce un cambio en sus estrategias competitivas, más orientadas hacia la diferenciación y la calidad del servicio, distanciándose del tradicional modelo basado en costes. El aumento en las innovaciones en producto y sobre todo en proceso se acompaña de cambios organizativos en la empresa y se asienta en experiencias de cooperación a lo largo de la cadena de valor y con las instituciones científicas. El estudio realizado también manifiesta que los trabajadores con niveles educativos más elevados muestran mayor capacidad para aprovechar de forma más eficiente el know-how de la empresa y traducir el conocimiento tácito en innovación.

Por último, en esta línea de trabajo vinculada a la innovación y la penetración de las TIC en la industria turística, merece citar el trabajo realizado por el observatorio de la Comisión europea e-BusinessW@ $\mathrm{tch}^{4}$, relativo a turismo ICT and eBusiness in the Tourism Industry (2006). El estudio indica que el turismo está en la vanguardia de la adopción de TIC y el e-negocio en el área de la e-comercialización y de ventas on-line. Sin embargo, este estudio revela que en el ranking de los 10 sectores estudiados en 2006, las cuentas de la industria del turismo solamente puntúan en el campo medio en relación con el uso total de las TIC y del e-negocio. Especialmente en relación con el despliegue de infraestructura TIC, las compañías turísticas todavía tienen una demora con respecto a sus contrapartes en otras industrias. El informe de la Comisión manifiesta que la capacidad de innovación en cuanto a producto y/o servicio es esencial para las compañías de turismo Europeas si quieren hacer frente a la competencia global. El 24\% de las empresas turísticas lanzaron en 2005 nuevos productos o servicios, de los cuales un 44\% estaban relacionados con el campo de las TIC. Un 19\% de las empresas turísticas introdujeron novedades en sus procesos internos (reservas on-line, etc.) de los cuales un 78\% eran gracias al aporte de las TIC. Los resultados del eBussinesW@tch, muestran que las expectativas de los clientes y la competitividad del mercado son los principales conduc-

4 La Comisión de las Comunidades Europeas lanzó el e-BusinessW@tch a finales de 2001 para supervisar adopción, desarrollo e impacto de las prácticas del negocio electrónico en diversos sectores de la economía en la Unión Europea. La iniciativa tiene su origen en los Planes de Acción de eEurope de 2002 y 2005. El Plan de Acción eEurope 2005 definió la meta "promover la puesta en marcha del e-negocio con el objetivo de aumentar la competitividad de las empresas Europeas y aumentar la productividad y el crecimiento de la inversión en tecnologías de la información y la comunicación, recursos humanos (notablemente las e-habilidades) y los nuevos modelos de negocio". El e-BusinessW@tch ha sido un instrumento importante para que la Comisión Europea determine el desarrollo y el progreso en este campo. 
tores del e-negocio en el sector del turismo. Enlazando con lo anteriormente dicho, en lo referente a la adopción de TIC y al tamaño de la compañía, en Europa el resultado más llamativo es que las compañías pequeñas son las más activas en el e-business, aunque la brecha entre las pequeñas y las grandes no es tan pronunciada como en otros sectores.

En cuanto a las barreras identificadas, parece que el tamaño pequeño de la mayoría de las compañías y los costes asociados a la adquisición de tecnologías constituyen las barreras principales del ebusiness en turismo. A ello se le suman las barreras asociadas a la complejidad de las tecnologías de comercio electrónico, los problemas de compatibilidad con tecnologías ya implantadas, el riesgo de seguridad y aspectos relativos a la privacidad, la percepción de aspectos legales sin resolver y la dificultad de encontrar un proveedor de servicios TIC de confianza.

Los estudios citados indican que las TIC permiten incorporar la innovación como un elemento de creación de valor en la actividad empresarial. De hecho, los procesos de innovación en estas empresas son más sofisticados e interdependientes y su uso les reporta una considerable ventaja competitiva. Las innovaciones formuladas afectan también al ámbito de competencia y modifican la manera en que los productores cubren las necesidades de los consumidores. En definitiva, en el nuevo entorno competitivo en el que se desarrolla la actividad turística mundial, parece que la adopción y el uso estratégico de las TIC pueden ayudar a la empresa a mejorar su eficiencia y a ser más competitiva, esencialmente a través del canal que supone el dinamismo innovador. Sin embargo, quedan barreras significativas que superar; obstáculos espinosos en una actividad económica globalizada que tiende hacia un ámbito de digitalización y uso intensivo de la información para el desarrollo de la cadena de valor turístico y en el que el tamaño o carencia de una masa crítica empresarial pueden ser fundamentales para determinar la competitividad empresarial y sectorial del mismo.

Siguiendo esta línea argumental, en este estudio se ha tratado de hacer un acercamiento a la realidad vasca con el objetivo de entender mejor cómo se está situando el sector turístico vasco en este nuevo entorno competitivo en el que se enmarca la actividad turística mundial. Para ello se ha realizado un estudio de cómo se está llevando a cabo esa adopción estratégica de innovación y de TIC, a la vez que trata de identificar cuáles son las principales barreras identificadas y que consecuentemente van a determinar las competitividad sectorial del sector turístico vasco.

\section{METODOLOGÍA}

En la línea de los estudios mencionados en el punto anterior, para este trabajo se han utilizado los datos de la encuesta "Cuestionario de necesidades tecnológicas del sector turístico en Euskadi"s. En dicho trabajo se trató de adaptar los contenidos de la defi-

\footnotetext{
5 Estudio realizado entre noviembre de 2004 y enero de 2005, gracias a un Convenio-Marco de colaboración suscrito entre la SPRI y TECNALIA y con la colaboración de la Viceconsejería de turismo, Dirección territorial de turismo y Dirección de promoción de turismo del Departamento de Industria, Comercio y Turismo del Gobierno Vasco.
} 
nición de innovación desarrollados, a las peculiaridades del sector turístico vasco. Siguiendo este esquema, se analizaban las empresas a través de 14 áreas de aplicación ${ }^{6}$ identificando herramientas, modelos, sistemas, o soluciones tecnológicas, definidas por cada área de innovación y se obtenía información relativa a la sistemática seguida por las empresas con el fin de incorporar innovación en sus organizaciones (medios más importantes de acceso a la tecnología punta o acceso a las nuevas formas de gestión). Por último se profundizaba en la percepción y auto-consideración que las organizaciones tenían de sí mismas y de su entorno en materia de innovación.

Para el presente trabajo el diseño del modelo empírico se ha ajustado al marco del Manual de Oslo ${ }^{7}$ que identifica las áreas de producto, de proceso (introducción de un nuevo, o significativamente mejorado, proceso de producción o distribución), de marketing (nuevos métodos de comercialización en materia de posicionamiento, de promoción, etc.) e innovación de organización, aunque se establecieron como punto de partida para el análisis exploratorio un conjunto de 49 variables, agrupadas a priori en ocho grupos. Es decir, el análisis exploratorio parte de la inclusión de variables pertenecientes a varios tipos de innovación: en producto, en proceso, en la comercialización-distribución, en marketing y comunicación, en organización y en innovación en TIC. Igualmente, en la línea de las pautas indicadas en este mismo manual, y en base al enfoque "sujeto" se han incluido dos factores como las fortalezas auto-percibidas en materia de innovación y las necesidades detectadas en materia de innovación.

La muestra para este estudio está compuesta por los casos de cuestionarios completados y recibidos por correo postal (el $24 \%$ de los 300 cuestionarios enviados) y las 59 entrevistas en profundidad, todos pertenecientes a empresas, agentes y organizaciones tanto públicas como privadas de C.A. de Euskadi. La muestra estratificada representa a 6 sectores principales de actividad turística: transporte, alojamiento, restauración, atracciones, agencias de viajes y gestores de destinos. La muestra además revela la presencia minoritaria de los grandes grupos y multinacionales en el sector turístico vasco y en referencia a su personalidad jurídica, las organizaciones de carácter privado aglutinan más de dos tercios de la selección de este trabajo.

A partir de dichos datos y teniendo en cuenta la demografía empresarial, en particular en lo referente al tamaño de la empresa, se ha llevado a cabo un análisis discriminante, entendido como el análisis más adecuado a la hora de comprender las diferencias entre grupos. Es decir, partiendo de la existencia de dos o más grupos bien definidos a

6 Innovación de Producto, Innovación de Proceso, Innovación de Comercialización, Innovación en Marketing, Innovación de Organización y Gestión, Innovación en TIC, Innovación en Seguridad, Control y Vigilancia, Innovación en Mantenimiento, Innovación medioambiental, Innovación en sensores, Innovación en Automática y Robótica, Innovación en Alimentación, Innovación en Limpieza y lavandería, Innovación en Jardinería, Otras innovaciones (deporte, congresos, act. cultural, etc.).

7 OCDE y Comunidades Europeas-Eurostat (2006): Manual de Oslo. Guía para la recogida e interpretación de datos sobre innovación, Tragsa.

8 El enfoque "sujeto" trata las actitudes y actividades innovadoras de la empresa en su conjunto, explorando los factores que influyen en el comportamiento innovador de la empresa y el ámbito de las diversas actividades de innovación, sus resultados y los efectos de la misma (OCDE, 2006: 28) 
priori (en este caso, según el número de empleados) se trata de describir las diferencias existentes entre esos grupos en base a los valores que toman ciertas variables. Igualmente, a través de este procedimiento se intenta clasificar nuevos organismos en alguno de los grupos preexistentes en función de los valores que toman ciertas variables

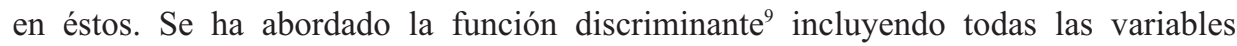
siguiendo el análisis estadístico de Wilks, que se utiliza principalmente para medir de forma secuencial el poder discriminatorio de cada una de las funciones que se van construyendo, empezando siempre por la primera (la de mayor capacidad discriminatoria), es decir un análisis según el método de selección de variables paso a paso. Acerca del último modelo empírico definido para el presente estudio queda exponer que se ha tenido que prescindir del constructo asociado a innovación de producto por errores técnicos vinculados a la naturaleza de la muestra. El modelo queda definido por los constructos correspondientes a: la innovación en proceso, innovación en marketing, innovación en organización y necesidades identificadas ${ }^{10}$.

Figura 1. Sectores a los que pertenecen los agentes de la muestra e Importancia de las áreas de innovación.

\section{EMPRESAS POR ACTIVIDAD}

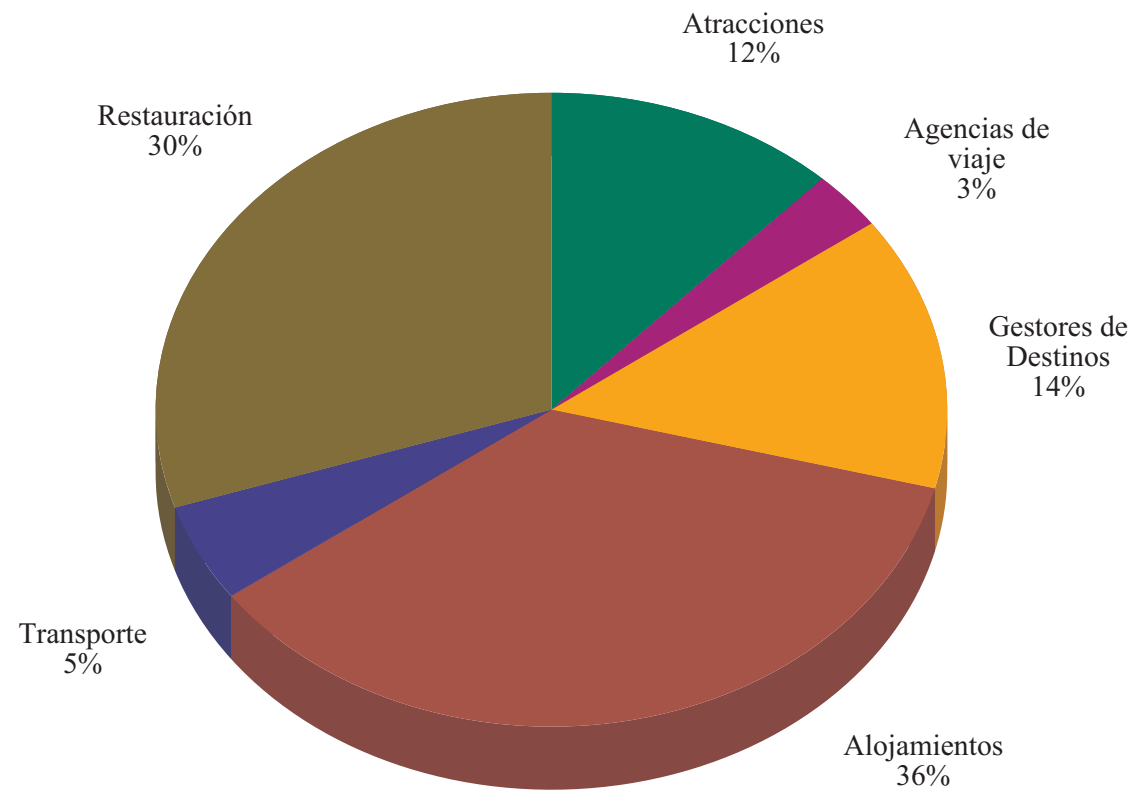

9 El número de funciones que pueden obtenerse es el mínimo entre el número de variables explicativas disponibles y el número de grupos menos uno. En este caso, al ser 2 los grupos en los que se clasifica la variable categórica y 39 las variables explicativas, se puede definir únicamente una función.

10 Los grupos introducidos, se corresponden además con aquellos, que realizando el análisis discriminante por pasos por grupos, alguna de las variables incluidas en los mismos ha resultado significativa. 
Figura 2. Sectores a los que pertenecen los agentes de la muestra e Importancia de las áreas de innovación.

IMPORTANCIA DE LAS ÁREAS DE INNOVACIÓN

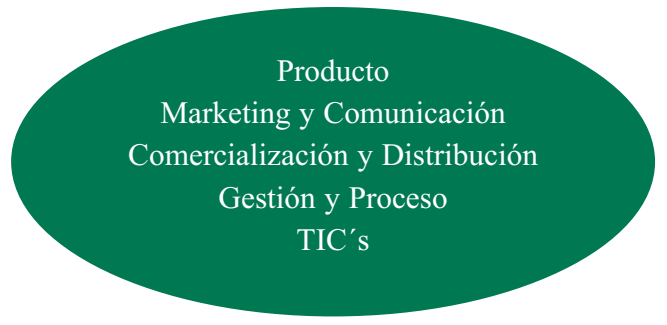

Medioambiente

Seguridad y control

Mantenimiento

Domótica, Robótica,

Mantenimiento, Alimentación,

Limpieza y Jardinería

\section{RESULTADOS}

A nivel descriptivo y haciendo hincapié en los resultados más importantes para este estudio $^{11}$, los resultados del análisis realizado revelan que el sector turístico vasco identifica la innovación de producto como esencial o importante para su supervivencia. Más de la mitad de los agentes consultados afirman haber ejecutado alguna iniciativa innovadora y un $65 \%$ muestra una disposición elevada para adquirir este tipo de servicios. En este sentido, los agentes coinciden en destacar el insuficiente aprovechamiento del patrimonio vasco natural y cultural desde el punto de vista turístico y destacan la necesidad de desarrollar contenidos y herramientas basadas en las nuevas tecnologías de la información y comunicación para hacer más atractivos y accesibles los nuevos productos turísticos.

El grado de importancia otorgada a la innovación y desarrollo de TIC en los procesos es muy elevado. Muestra de ello es que más de la mitad del sector consultado está llevando a cabo innovación en la gestión de producción y en gestión de proveedores. En cuanto a la innovación en la comercialización, ésta sea los sistemas de gestión de reservas (CRS, GDS), los gestores de viajes automáticos o el comercio on-line de los productos turísticos, lo que muestra el interés principal por la gestión de reservas, autoaprendiazaje o el comercio on-line.

11 Pueden verse los resultados descriptivos de este estudio en Alzua, A. et al., (2006): Innovación en el Sector Turístico Vasco. VI Congreso Nacional "Turismo Tecnologías de la Información y Comunicaciones" TuriTec 2006 [pp. 167-184] Universidad de Málaga, 18-20 de Octubre de 2006) 
La innovación en las áreas propias del marketing han sido valoradas y priorizadas frente a las demás ya referidas, dando especial importancia a las diferentes formas de acercarse a un servicio personalizado, que adapte la demanda al cliente, dejando en un segundo plano temas como los saludos y recordatorios (más específico de algunos subsectores). Por otro lado, respecto a la innovación de organización los resultados expresan que el sector turístico vasco está interesado en los sistemas de planificación estratégica, los sistemas de calidad y los de motivación del personal.

Coincidiendo con las principales áreas identificadas por el Manual de Oslo ${ }^{12}$ en el resto de las secciones presentadas en su momento, y mucho más específicas (Innovación en seguridad, Innovación en mantenimiento, Innovación medioambiental, Innovación en sensores o Innovación en Automática y Robótica entre otras), se observa una disminución del número de respuestas recogidas.

\subsection{ANÁLISIS DISCRIMINANTE}

En base a la metodología diseñada y a las reflexiones teóricas sobre la incidencia del tamaño de la empresa en la actividad innovadora de las mismas, se ha optado por utilizar como variable dependiente en el análisis discriminante, el tamaño de la empresa, partiendo de 2 grupos: el Grupo 1, que se correspondería con empresas pequeñas $(<9$ empleados) o el Grupo 2 con más de 10 empleados. En ambos casos, se ha tomado el número de empleados fijos en las mismas.

Figura 3. Distribuciones de la muestra según el Número de empleados fijos.

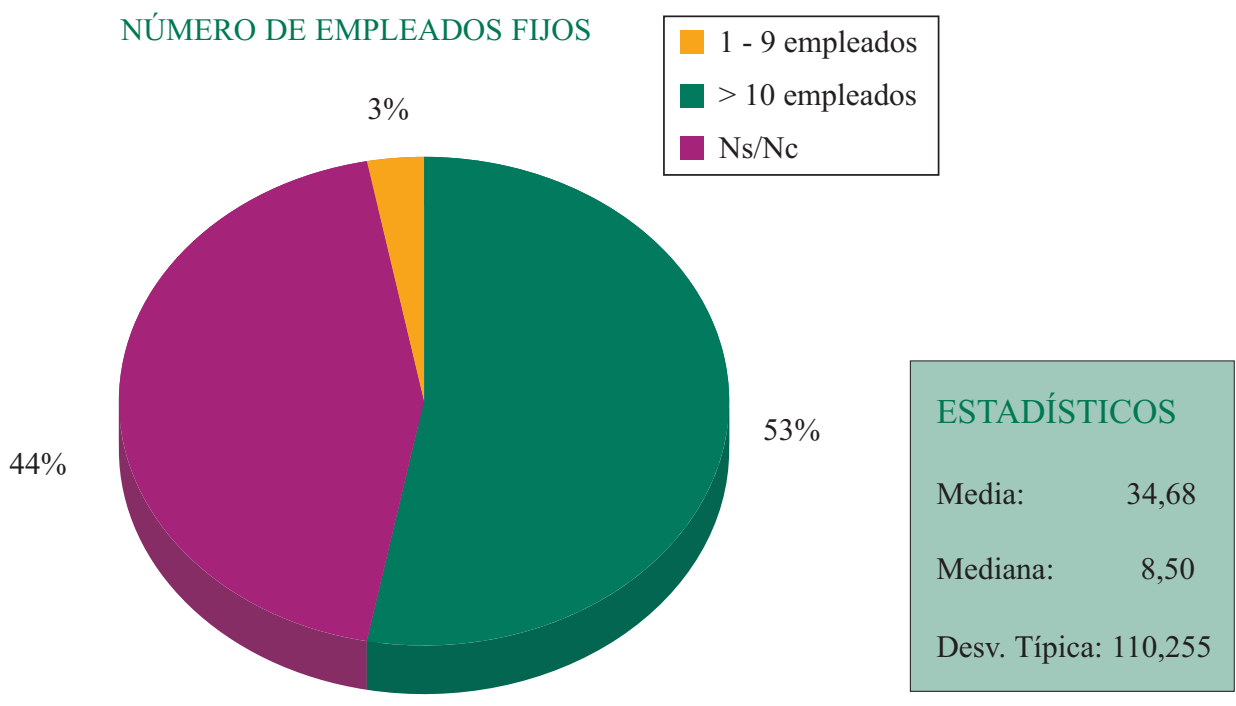

12 OCDE y Comunidades Eusropeas-Erostat (2006). Manual de Oslo. Guía para la recogida e interpretación de datos sobre innovación. Tragsa. 
Con el análisis discriminante se procura, en primer lugar, describir las diferencias existentes entre los dos grupos en base a los valores que toman las innovaciones sobre las empresas e instituciones identificadas y en segundo lugar, una vez identificadas las innovaciones explicativas, determinar cuáles de éstas son las que más contribuyen a comprender las divergencias entre grupos. Con este objetivo primeramente se ha realizado una comparativa del valor medio y la variabilidad de cada variable dentro de los dos grupos y posteriormente se ha procedido con un análisis discriminante univariante, es decir, con los correspondientes contrastes F univariantes de igualdad de medias entre los grupos para cada una de las variables consideradas (véanse ANEXOS I y II).

Los niveles de significación del contraste $\mathrm{F}$ para igualdad de medias entre los dos grupos (valor estadístico F que aparece en la tabla 1), junto con los correspondientes niveles de significación indican que las variables de Innovación en proceso, Innovación en Marketing, Innovación en Organización y la Necesidad de encontrar y movilizar RRHH (E5b), esta última como variable de necesidades detectadas en materia de innovación, son las que cuentan con un potencial mayor de discriminación.

Tabla 1. Contrastes univariantes de igualdad de medias entre los grupos con valores significativos

\begin{tabular}{llrrrrr} 
& & Lambda & & & & \\
Q2a2 & Proceso- Gestión de producción asistida &, 901 & 7,246 & 1 & 66 &, 009 \\
\hline Q2b2 & Proceso- Sist. Gestión proveedores &, 807 & 15,757 & 1 & 66 &, 000 \\
\hline Q2c2 & Proceso- Integración sist gestión &, 940 & 4,246 & 1 & 66 &, 043 \\
\hline Q2d2 & Proceso- Automatización, robotización &, 929 & 5,074 & 1 & 66 &, 028 \\
\hline Q2f2 & Proceso-Técnicas control, medida proceso &, 944 & 3,933 & 1 & 66 &, $052^{*}$ \\
\hline Q3c2 & Comercialización- Comercio on-line &, 954 & 3,172 & 1 & 66 &, $080^{*}$ \\
\hline Q4d2 & Marketing-Sist gestión sugerencias y & & & & & \\
& reclamaciones &, 894 & 7,793 & 1 & 66 &, 007 \\
\hline Q4e2 & Marketing-Sist segmentación clientes &, 852 & 11,446 & 1 & 66 &, 001 \\
\hline Q5a2 & Organización- Vigilancia de mercado &, 939 & 4,322 & 1 & 66 &, 042 \\
\hline Q5d2 & Organización-Sist calidad &, 945 & 3,875 & 1 & 66 &, $053^{*}$ \\
\hline Q5e2 & Organización-Sist autoformación, on-line &, 943 & 4,022 & 1 & 66 &, 049 \\
\hline E5a & Necesidad-Acceder a mercados innovadores &, 958 & 2,905 & 1 & 66 &, $093^{*}$ \\
\hline E5b & Necesidad- Encontrar y movilizar RRHH &, 917 & 5,973 & 1 & 66 &, 017 \\
\hline
\end{tabular}

De hecho se puede decir que la variable Disposición de sistemas de gestión de proveedores (Q2b2), es la que más discrimina entre los dos grupos a nivel individual. Los resultados correspondientes a la estimación de la función discriminante (según método de selección de variables paso a paso con los niveles de significación acotados), garantizando así unos valores mínimos para $\mathrm{F}$, después de 56 etapas, finaliza con una selección de 2 variables de entre las 22 disponibles (Tabla 2). 
Tabla 2. Variables discriminantes significativas

\begin{tabular}{llccc} 
& & Lambda de Wilks & F exacta & Sig. \\
\hline Q2b2 & Proceso- Sist. Gestión proveedores &, 807 & 15,757 &, 000 \\
\hline Q4e2 & Sist. De segmentación de clientes &, 741 & 11,354 &, 000 \\
\hline
\end{tabular}

Los datos muestran que la primera variable que entra en el modelo es Disposición de sistemas de gestión de proveedores (Q2b2) que, como ya se anticipaba, es la que a nivel individual conseguía una mayor discriminación según el criterio Lambda de Wilks e igualmente, en el segundo paso se incorpora la variable Disposición de sistemas de segmentación de clientes (Q4e2). Esto indica que de todos los posibles emparejamientos entre las 23 variables con las que se ha trabajado, éstas dos mencionadas son las que mayor capacidad tienen de separar las empresas en función de su tamaño (si tienen menos o más de 10 empleados). La tabla 3 recoge los coeficientes que tienen las 2 variables seleccionadas en la función discriminante estimada junto al término constante, definiendo la función discriminante resultante como:

$$
Y 1=-1,749+1,926 Q 2 b 2+1,360 Q 4 e 2
$$

Tabla 3. Coeficiente de las funciones discriminantes canónicas

\begin{tabular}{llc} 
& & Función \\
\hline Q2b2 & Proceso- Sist. Gestión proveedores & 1,926 \\
\hline Constante & Sist. De segmentación de clientes & 1,360 \\
\hline
\end{tabular}

En la tabla 4 se encuentran los valores del contraste de significación secuencial basado en el estadístico lambda de Wilks, que indican si es significativa.

Tablas 4. Estadístico Lambda de Wilks

\begin{tabular}{ccccc} 
Contraste de las funciones & Lambda de Wilks & Chi-cuadrado & gl & Sig. \\
\hline 1 &, 741 & 19,476 & 2 &, 000 \\
\hline
\end{tabular}

Para la interpretación de los resultados obtenidos, se analizan en primer lugar, las posiciones relativas de los distintos casos en el espacio definido por la función discriminante, como la manera de saber cuáles son finalmente las que más contribuyen a la discriminación entre los dos grupos. El valor de referencia van a ser los centroides de los dos grupos, a partir de las puntuaciones discriminantes (Tabla 5), ya que el análisis de la posición relativa de dichos centroides en el eje discriminante permite una perspectiva global de la separación entre los grupos que puede atribuirse a la función discriminante. 
Tabla 5. Coordenadas de los centroides en la función discriminante

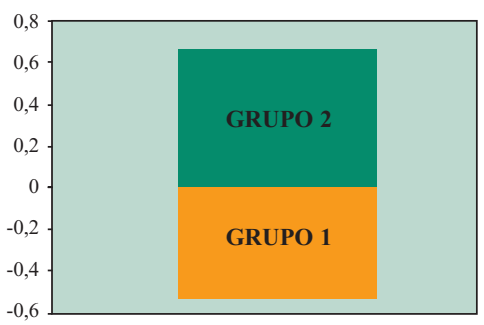

\begin{tabular}{lcc} 
& $\mathrm{N}^{\mathrm{o}}$ de empleados & Función \\
GRUPO 1 & $1-9$ empleados &,- 553 \\
\hline GRUPO 2 & $>10$ empleados &, 636 \\
\hline
\end{tabular}

Tabla 6. Medidas interpretativas de las funciones discriminantes

\begin{tabular}{llcc} 
& Q2b2 & $\begin{array}{c}\text { Coeficientes } \\
\text { estandarizados } \\
\text { Función 1 }\end{array}$ & $\begin{array}{c}\text { Coeficientes de } \\
\text { estructura } \\
\text { Función 1 }\end{array}$ \\
\hline Q4e2 & Sist. segmentación clientes & $\mathbf{, 7 2 2}$ & $\mathbf{8 2 7}$ \\
\hline Q2c2 & Integración sistemas de gestión & $\mathbf{, 5 7 2}$ &, $\mathbf{7 0 5}$ \\
\hline Q5e2 & Sist. autoformación, on-line &, 454 \\
\hline Q4b2 & Sist. saludos y recordatorios &, 385 \\
\hline Q5d2 & Sistemas de calidad &, 377 \\
\hline Q5a2 & Vigilancia de mercado &, 375 \\
\hline Q4d2 & Sist. gestión sugerencias y reclamaciones &, 340 \\
\hline Q5c2 & Sist. yield management &, 290 \\
\hline E5d & Identificar nuevas tecnologías &, 266 \\
\hline Q5b2 & Sist. planificación estratégica &,- 265 \\
\hline Q2a2 & Gestión de producción asistida &, 248 \\
\hline Q4c2 & Sist. información personalizada &, 220 \\
\hline Q5f2 & Sist. motivación de personal &, 207 \\
\hline Q2f2 & Técnicas de control, medida y proceso &, 175 \\
\hline E5c & Encontrar y movilizar RR Financieros &, 155 \\
\hline Q2d2 & Automatización, robotización &,- 147 \\
\hline Q4a2 & Sist. imágenes y videos &, 118 \\
\hline E5a & Acceder a mercados innovadores &, 101 \\
\hline E5b & Encontrar y movilizar RRHH &,- 042 \\
\hline Q2e2 & Domótica &, 026 \\
\hline E5e & Compartir conocimiento, cooperación &, 012 \\
\hline & &,- 010 \\
\hline
\end{tabular}

En este caso se comprueba que son extremos opuestos, por lo que la separación entre ambos grupos queda contrastada, sin embargo ese análisis no ofrece una base para 
explicar las diferencias en términos de las variables independientes. Para ello se recurre a los coeficientes estandarizados de las 2 variables incluidas en las funciones discriminantes y las correlaciones entre las 23 variables disponibles y la función discriminante estimada (los coeficientes de estructura).

La primera columna detalla las contribuciones de las 2 variables seleccionadas a cada función de forma individual, con los valores superiores en el coeficiente estandarizado, lo que indica (independientemente del signo) que continúan siendo las variables que más diferencian los dos grupos.

La segunda columna permite interpretar los resultados y analizar en qué sentido influyen las variables anteriores en las diferencias entre los grupos. A través de los valores de la segunda columna, los coeficientes de estructura ${ }^{13}$, calculados para todas las variables disponibles, incluso para las 21 que no forman parte de la función discriminante y que permiten determinar qué variables correlacionan más con la función discriminante a través de coeficientes de estructura elevados. Por otro lado, se ha ido viendo como la primera función discriminante, separa bien los grupos asociando el Grupo 1 (empresas de entre 1 y 9 empleados) con valores menores o negativos (centroide: -553) y por lo tanto se corresponde con las variables con signo negativo mientras que el Grupo 2 (empresas de 10 o más empleados) con valores positivos (centroide: 636) y consecuentemente con las variables con coeficiente de estructura positivo, que tendrán una correlación positiva con el mismo.

Los mayores pesos se identifican en la Disposición de sistemas de gestión de proveedores $(, 827)$ y la Disposición de sistemas de segmentación clientes $(, 705)$, seguido por la Disposición de integración de sistemas de gestión $(, 454)$ con valores muy inferiores así como por la Disposición de sist. autoformación, on-line (,385) de sist. de saludos y recordatorios $(, 377)$, de sistemas de calidad $(, 375) \mathrm{y}$ de sistemas de vigilancia de mercado, todos ellos con valores por encima de los 300. Con valores ya más bajos aparecerían la Disposición de sist. de gestión sugerencias y reclamaciones $(, 290)$, sistemas de yield management (,266), la necesidad de identificar nuevas tecnologías $(-, 265)$ todos ellos por encima de 0,250 .

\section{CONCLUSIONES}

La primera conclusión que se extrae es que, en este estudio, la función discriminante resultante del modelo identificado (innovación en proceso, en marketing, en organización y las necesidades detectadas) identifica el tamaño de la organización como clave para poder abordar innovaciones como la gestión de proveedores y la segmentación de clientes. Procesos, ambos, que impactan directamente en la capacidad y la mayor eficiencia empresarial.

13 Estas variables no entraron en el modelo, bien porque no mejoraban la discriminación o porque estaban muy correlacionadas con otra variable incluida previamente y resultaban redundantes, lo que no significa necesariamente que no cuenten con un efecto sustancial en la interpretación del eje discriminante, sino que permiten determinar qué variables correlacionan más con la función discriminante a través de coeficientes de estructura elevados. 
Siguiendo la línea argumental del marco teórico, parece que la intensificación en el uso de las TIC redunda en importantes aumentos de la productividad (factor trabajo), y como la innovación en las empresas turísticas depende positivamente de la intensificación de los usos TIC, de la cooperación con otras empresas y de la calificación del trabajo, mientras la productividad del trabajo depende directamente del rendimiento del capital invertido así como también del uso de las TIC. De los resultados se desprende que la innovación en las empresas más grandes, de 10 o más empleados (frente a las de menos), se caracteriza por disponer de herramientas centradas en mejorar su eficacia en la relación con otros elementos importantes de la cadena de valor, es decir, por disponer de sistemas centrados en la gestión de proveedores y en la segmentación clientes.

En la misma línea, las empresas de mayor tamaño se caracterizan por integrar sistemas de gestión y en menor medida, se caracterizan por innovar en organización como disponer de sistemas de calidad o de vigilancia de mercados además de en marketing directo con los clientes a través de sistemas de saludos y recordatorios o de sistemas de gestión de sugerencias y reclamaciones. Estos resultados encajan con lo anteriormente dicho, donde se relaciona la mejora de productividad y la intensificación en el uso de las TIC con la necesidad de que estos cambios vengan acompañados por un esfuerzo de cambio organizativo, en el cual se adopte una actitud proactiva y no reactiva en relación a la innovación y que promueve la flexibilidad del trabajo y del conjunto de la organización.

Parece fundamental que la intensificación en el uso de las TIC venga acompañada de un cambio organizativo y en paralelo a nuevas estrategias de externalización o cooperación de las actividades más cercanas a la incorporación de innovaciones tecnológicas. Por lo tanto, se deberían fomentar las políticas de cooperación entre las empresas y las instituciones científicas, con el fin de crear un sistema de innovación (local, regional o nacional) para la actividad turística, que estimulara el desarrollo. A este modelo deben sumarse las empresas más pequeñas (micropymes) ya que como indican los resultados de este estudio, las empresas de menos de 10 empleados, no se caracterizan tanto por disponer o centrar su interés en un tipo de herramientas o de sistemas, si no, en que identifican una serie de necesidades comunes en materia de innovación, como son la necesidad de identificar y utilizar nuevas tecnologías, el encontrar y movilizar recursos financieros para mejorar su competitividad basada en la innovación.

Pudiera ser que la dificultad de generar resultados más concretos se deba a la heterogeneidad del universo de empresas del sector turístico por lo que sería importante poder pormenorizar el análisis de la innovación de manera subsectorial. Sería recomendable que futuros estudios abordaran estas cuestiones con muestras significativas para cada subsector turístico: hoteleros, agencias de viaje, gestores de destino, etc. En este sentido, hay que prestar importancia a la naturaleza de cada subsector, su capacidad de gestión, la capacidad de innovación y la capacidad de internacionalización de las mismas, ya que la mejora empresarial de las mismas y, consecuentemente la competitividad del sector, depende directamente de la cantidad y calidad de los recursos internos de la empresa (recursos humanos, recursos físicos y financieros). Así mismo, se debe prestar especial atención a los recursos externos que le proporciona su entorno económico 
(redes de cooperación, acceso a la tecnología, acceso a mercados, proveedores eficientes y servicios avanzados) y consecuentemente difieren en su capacidad para aprovecharse de los recursos del entorno.

\section{BIBLIOGRAFÍA}

Alzua, A., Abad, M. y Oyón, C. (2006): “Innovación en el Sector Turístico Vasco”, Actas del VI Congreso Nacional "Turismo Tecnologías de la Información y Comunicación TuriTec 2006, pp. 167-184, Universidad de Málaga.

Amin, A. \& Thrift, N. (2002): Cities. Reimagining the Urban, Blackwell Publishers.

Audretsch, D.; Callejón, M. y Aranguren, M.J. (2003): "Entrepreneurship, small firms and self-employment", Small Business Economy.

Blanke, J. y Chiesa, T. (ed.) (2007): The travel \& tourism competitiveness report 2007: furthering the process of economic development, Geneva, World Economic Forum.

Buhalis, D. y O'Connor, P. (2006): Tourism Management Dynamics. Trends, management and tools, Oxford, Elsevier Butterworth-Heinemann.

Buhalis, D. (2003): eTourism information technology for strategic tourism management. Prentice Hall, Financial Times.

Calderón Vázquez, F.J. (2007): Distrito turístico rural un modelo teórico desde la perspectiva de la oferta, Edición electrónica gratuita. [Texto completo en www.eumed.net/tesis/2007/fjcv/].

Castells, M. (2000): The Rise of the Network Society, Blackwell Publishers

Castells, M. (1997): La Era de la información: economía, sociedad y cultura, Madrid, Alianza, 3v.

Comisión Europea (1995): Libro Verde de la Innovación. ES/13/95/55220800.P00 (FR)

Decelle, X. (2004): A conceptual and dynamic approach to innovation in tourism en Innovation and Growth in Tourism: Conference Papers, OCDE. Swiss State Secretariat for Economic Affairs, Lugano, Suiza, 18-19 Septiembre de 2003, 16 pp.

Donaire, J.A. (1998): “La reconstrucción de los espacios turísticos. La geografía del turismo después del fordismo" en Sociedade e Territorio, 28. Porto Alegre

Dwyer, L. Y kim, C. (2003): Destination Competitiveness: Determiannts ans Indicators. Current Issues in ourism, 6 (5): 369-414.

European Commission (2006): ebusiness-watch. ICT and e-Business in the Tourism Industry. ICT adoption and e-business activity in 2006. Sector report 8/2006

http://www.ebusiness-watch.org/studies/sectors/tourism/documents/Tourism_2006.pdf

Evangelista, R. y Savona, M. (2003): Innovation, employment and skills in services. Firm and sectoral evidence, Structural Change and Economic Dynamics, 14 (4): 449-474.

Garay Tamajón, L. A. y Ammetller Montes, G. (2004): Innovación y nuevas estrategias competitivas en el sector de servicios menos intensivos en conocimiento: la industria turística, el comercio y el transporte. I Jornades d'Economia del Turismo. 
Gobierno Vasco (2007): Plan de competitividad del turismo vasco 2006-2009. Documentos del Turismo Vasco, 18. Vitoria-Gasteiz, Servicio Central de Publicaciones del Gobierno Vasco.

González, J.L. y Peña, I., (2007): Determinantes de la Capacidad de Innovación de los Negocios Emprendedores en España”. Economía Industrial, 363: 129-147.

Hannam, K.; Sheller, M. y Urry, J. (2006): Mobilities, Immobilities and Moorings. Mobilities 1(1), 1-22.

Instituto de Estudios Turísticos (2006): Ocupación Turismo Rural en $\mathrm{http} / /$ www.iet.tourspain.es/paginas/EOTR.aspx?seleccion=tablas\&periodo=mensual\&idioma=es-ES\&option=otr\&option2=EOTR consultado en octubre 2006.

Molero, J. y Valadez, P. (2005): "Factores determinantes de la competitividad de los servicios: la importancia de la innovación”, Información Comercial Española, 824: Julio- Agosto.

Mora Sánchez, A., Lanquar, R., Martí, F.P. (1999): Los efectos de la innovación tecnológica en el turismo. I Congreso Nacional de Turismo y Tecnologías de la Información (TURITEC).

Morales, L., Peña, I., (2003): “Dinamismo de Nuevas Empresas y Clusters Naturales: Evidencia de la CAPV, 1993-1999”. Ekonomiaz, Revista Vasca de Economía 53: 160-183.

OCDE y Comunidades Europeas-Eurostat (2006): Manual de Oslo. Guía para la recogida e interpretación de datos sobre innovación, Tragsa.

Requena et al. (2007): “Tecnologías de la Información y Comunicación, Innovación y Actividad Turística: hacia la empresa en red”, Cuadernos de Turismo, 19, 217-240.

Sánchez Rivero, M. (2006): "Elaboración de un ranking de competitividad de los destinos turísticos españoles: un análisis provincial mediante modelos de estructura latente", Revista de análisis turístico, 1: 4-23.

Sheller, M. y Urry, P.J. (2006): Mobile technologies of the city. Routledge.

Taberner, J. (2006): "Proposal of two complementary indexes for measuring tourism competitiveness of Mediterranean destinations: outcoming results of a pilot study from the demand side", 4th International Doctoral Tourism and leisure colloquium. Barcelona 2006.

Urry, J. (2000): Sociology beyond societies. Mobilities for the twenty-first century, London, Routledge.

Urry, J. (1995): Consuming places, London, Routledge.

Vilaseca, J., Torrent, J., Meseguer y Rodriguez, I. (2007): “An integrated model of adoption and development of e-commerce in companies", International Advances in Economic Research, Vol. 13 (2).

Vilaseca, J., Torrent, J., Lladós, J. y Garay, L. (2006): “TIC, Innovación y Productividad en la empresa turística catalana", Actas del VI Congreso Nacional "Turismo Tecnologías de la Información y Comunicación TuriTec 2006. Universidad de Málaga.

VVAA (2007): Tema de portada: Turismo. Índice. Revista de Estadística y Sociedad, 24 (Septiembre 2007), Madrid, Universidad Autónoma de Madrid. 
ANEXO I: Medias y desviaciones típicas por grupos y total

\begin{tabular}{|c|c|c|c|c|c|c|}
\hline & \multicolumn{3}{|c|}{ MEDIA } & \multicolumn{3}{|c|}{ Desviación típica } \\
\hline & $<10$ & $>10$ & TOTAL & $<10$ & $>10$ & TOTAL \\
\hline & \multicolumn{3}{|c|}{ empleados empleados } & \multicolumn{3}{|c|}{ empleados empleados } \\
\hline Q1a2 - Nuevos productos turísticos &, 489 &, 594 &, 537 &, 4930 &, 4281 & ,4641 \\
\hline Q1b2- Nuevas actividades complementarias & ,416 &, 542 & ,474 & ,4638 & ,4162 &, 4440 \\
\hline Q2a2-Gestión de producción asistida &, 590 & ,838 & ,703 & ,4312 & ,3039 & ,3959 \\
\hline Q2b2-Sist. Gestión proveedores & ,438 &, 800 & 603 & ,4159 &, 3183 & ,4139 \\
\hline Q2c2-Integración sist gestión & ,372 &, 574 &, 464 & ,3960 & ,4117 &, 4129 \\
\hline Q2d2-Automatización, robotización & 219 & ,406 & ,304 &, 2767 & ,4066 &, 3522 \\
\hline Q2e2-Domótica &, 103 &, 173 &, 135 &, 1685 &, 2841 & ,2294 \\
\hline Q2f2-Técnicas control, medida proceso & ,289 & ,450 &, 362 & ,3072 &, 3622 & ,3406 \\
\hline Q3a2-Sis gestión reservas & ,480 & 608 &, 539 & ,4714 & .4341 &, 4560 \\
\hline Q3b2-Gestores viajes automáticos &, 158 &, 239 &, 195 & ,2696 & ,3096 & ,2892 \\
\hline Q3c2-Comercio on-line & ,439 & ,636 &, 529 & ,4602 & ,4446 & ,4606 \\
\hline Q4a2-Sist imágenes y videos & ,675 &, 509 & 600 & ,4381 &, 4839 & ,4636 \\
\hline Q4b2-Sist saludos y recordatorios & ,249 & ,361 &, 300 & ,3627 & ,4073 & ,3849 \\
\hline Q4c2-Sist información personalizada & ,416 &, 513 & ,460 & ,4637 & ,4742 & ,4676 \\
\hline Q4d2-Sist gestión sugerencias y reclamacio & ,415 & ,773 &, 578 & ,4719 &, 5841 &, 5519 \\
\hline Q4e2-Sist segmentación clientes & 274 & 621 & ,432 & ,3954 & ,4495 &, 4525 \\
\hline Q5a2-Vigilancia de mercado & 200 &, 403 & ,292 & ,3431 & ,4623 &, 4115 \\
\hline Q5b2-Sist planificación estratégica & 469 &, 571 &, 516 & ,4158 & ,4416 & ,4276 \\
\hline Q5c2-Sist yield management &, 113 & ,202 &, 154 &, 1675 &, 3202 & ,2509 \\
\hline Q5d2-Sist calidad &, 340 &, 562 &, 441 & ,4356 & ,4962 &, 4740 \\
\hline Q5e2-Sist autoformación, on-line & ,291 &, 509 & ,390 & ,4068 & ,4923 & ,4577 \\
\hline Q5f2-Sist motivación de personal & ,426 &, 439 &, 432 & ,4199 & ,4531 & ,4321 \\
\hline Q6b2-Intranet & ,603 & ,650 & 624 & ,4258 & ,4430 & ,4311 \\
\hline Q6c2-Extranet & ,456 & ,444 &, 451 & ,4237 & ,3849 & ,4035 \\
\hline Q6d2-Conexión internet & ,94 &, 90 & ,92 &, 246 & ,305 & 275 \\
\hline E3a-Cooperac con proveedores, asociaciones &, 529 &, 571 &, 523 & ,4555 & ,4913 & ,4686 \\
\hline E3b-Contar con personal cualificado & ,481 &, 583 &, 527 & ,4564 & ,4844 & ,4686 \\
\hline E3c-Adaptabilidad a la producción y mercado & ,386 & ,279 & ,338 & ,4481 &, 4396 & ,4442 \\
\hline E3d-Método producción óptima & ,076 &, 169 &, 118 & ,2285 & ,3717 & ,3035 \\
\hline E3e-Lider en explorar experiencias mercado &, 112 &, 172 &, 139 &, 2750 & ,3714 &, 3214 \\
\hline E3f-Progresos tecnológicos en I+D &, 060 &, 002 & 034 & ,2281 &, 0082 &, 1698 \\
\hline E5a-Acceder a mercados innovadores & ,496 &, 312 & ,413 & ,4503 & ,4353 & ,4498 \\
\hline E5b- Encontrar y movilizar RRHH & 130 & ,352 & 231 & ,2782 & ,4607 &, 3860 \\
\hline E5c- Encontrar y movilizar RR Financieros & ,377 &, 370 &, 374 & ,4355 & ,4581 & ,4426 \\
\hline E5d-Identificar nuevas tecnologías & ,446 &, 411 & ,430 & ,4464 & ,4663 & ,4524 \\
\hline E5e-Compartir conocimiento, cooperación & 239 &, 158 & 231 & ,4166 & ,3381 & 3860 \\
\hline
\end{tabular}


ANEXO II: Contrastes univariantes de igualdad de medias entre los grupos

\begin{tabular}{|c|c|c|c|c|c|}
\hline & Lambda de Wilks & $\mathrm{F}$ & gl1 & $\mathrm{g} 12$ & Sig. \\
\hline SMEAN(Q1a2) & ,987 &, 851 & 1 & 66 &, 360 \\
\hline SMEAN(Q1b2) & ,980 & 1,366 & 1 & 66 & ,247 \\
\hline SMEAN(Q2a2) & 901 & 7,246 & 1 & 66 &, 009 \\
\hline SMEAN(Q2b2) & ,807 & 15,757 & 1 & 66 &, 000 \\
\hline SMEAN(Q2c2) & ,940 & 4,246 & 1 & 66 &, 043 \\
\hline SMEAN(Q2d2) & ,929 & 5,074 & 1 & 66 & ,028 \\
\hline SMEAN(Q2e2) & ,976 & 1,590 & 1 & 66 & ,212 \\
\hline SMEAN(Q2f2) & ,944 & 3,933 & 1 & 66 &, 052 \\
\hline SMEAN(Q3a2) & ,980 & 1,346 & 1 & 66 &, 250 \\
\hline SMEAN(Q3b2) & ,980 & 1,334 & 1 & 66 & ,252 \\
\hline SMEAN(Q3c2) & ,954 & 3,172 & 1 & 66 &, 080 \\
\hline SMEAN(Q4a2) & ,968 & 2,198 & 1 & 66 &, 143 \\
\hline SMEAN(Q4b2) &, 978 & 1,454 & 1 & 66 & ,232 \\
\hline SMEAN(Q4c2) & ,989 & ,728 & 1 & 66 &, 396 \\
\hline SMEAN(Q4d2) &, 894 & 7,793 & 1 & 66 &, 007 \\
\hline SMEAN(Q4e2) &, 852 & 11,446 & 1 & 66 &, 001 \\
\hline SMEAN(Q5a2) & ,939 & 4,322 & 1 & 66 &, 042 \\
\hline SMEAN(Q5b2) & ,986 & ,962 & 1 & 66 &, 330 \\
\hline SMEAN(Q5c2) & 968 & 2,175 & 1 & 66 &, 145 \\
\hline SMEAN(Q5d2) & ,945 & 3,875 & 1 & 66 & ,053 \\
\hline SMEAN(Q5e2) & ,943 & 4,022 & 1 & 66 &, 049 \\
\hline SMEAN(Q5f2) & 1,000 &, 014 & 1 & 66 & ,906 \\
\hline SMEAN(Q6b2) & ,997 & ,201 & 1 & 66 &, 655 \\
\hline SMEAN(Q6c2) & 1,000 &, 015 & 1 & 66 & ,904 \\
\hline Q6 &, 995 & ,286 & 1 & 66 &, 595 \\
\hline SMEAN(E3a) & 1,000 & ,011 & 1 & 66 & ,918 \\
\hline SMEAN(E3b) & ,988 & ,806 & 1 & 66 &, 373 \\
\hline SMEAN(E3c) & ,985 & ,981 & 1 & 66 &, 326 \\
\hline SMEAN(E3d) & ,976 & 1,596 & 1 & 66 & ,211 \\
\hline SMEAN(E3e) & ,991 &, 580 & 1 & 66 & ,449 \\
\hline SMEAN(E3f) & ,970 & 2,007 & 1 & 66 & ,161 \\
\hline SMEAN(E5a) & ,958 & 2,905 & 1 & 66 & ,093 \\
\hline SMEAN(E5b) & ,917 & 5,973 & 1 & 66 &, 017 \\
\hline SMEAN(E5c) & 1,000 &, 004 & 1 & 66 &, 950 \\
\hline SMEAN(E5d) & ,999 & ,098 & 1 & 66 &, 755 \\
\hline SMEAN(E5e) & ,970 & 2,068 & 1 & 66 & , 155 \\
\hline
\end{tabular}

